\title{
The role of the dorsal striatum and dorsal hippocampus in probabilistic and deterministic odor discrimination tasks
}

\author{
Hisham E. Atallah, Jerry W. Rudy, ${ }^{1}$ and Randall C. O'Reilly \\ Department of Psychology and Center for Neuroscience, University of Colorado, Boulder, Colorado 80302, USA
}

\begin{abstract}
Three experiments explored the contribution of the cortico-striatal system and the hippocampus system to the acquisition of solutions to simultaneous instrumental odor discriminations. Inactivation of the dorsal striatum after rats had reached criterion on a three problem probabilistic set of discriminations-A $(80 \%)$ vs. B $(20 \%), C(67 \%)$ vs. $D(33 \%), E(67 \%)$ vs. $F(33 \%)$-impaired test performance and disrupted performance when the rats were tested with novel cue combinations ( $C$ vs. $F$ and $E$ vs. D), where control animals chose $C$ and $F$. In contrast, inactivating the dorsal hippocampus enhanced performance on this task and on a deterministic discrimination A $(100 \%)$ vs. B (0\%). These results are consistent with the complementary learning systems view, which assumes that the cortico-striatal and hippocampal system capture information in parallel. How this information combines to influence task performance depends on the compatibility of the content captured by each system. These results suggest that the trial-specific information captured by the hippocampal system can be incompatible with the across-trial integration of trial outcomes captured by the cortico-striatal system.
\end{abstract}

There are both experimental and theoretical reasons to believe that the memory system supported by cortico-striatal areas and the system supported by the hippocampal formation have very different properties (Hirsh 1974; Oberg and Divac 1975; Mishkin and Petrie 1984; Mishkin et al. 1984; White and McDonald 2002). The cortico-striatal system is believed to support instrumental behaviors that are modified by the outcomes or rewards that they produce. This system integrates the outcomes of multiple response-reward experiences. In contrast, the hippocampal formation is part of the episodic memory system that is designed to capture representations of individual experiences and keep them separate (see Teyler and DiScenna 1986; Tulving and Markowitsch 1998; O'Reilly and Rudy 2001; Teyler and Rudy 2007). Thus, these two systems have complementary functions (Atallah et al. 2004).

Given that these systems are normally both online, their complementary functions (Mishkin and Petrie 1984; Mishkin et al. 1984; Sherry and Schacter 1987; McClelland et al. 1995; White and McDonald 2002) might sometimes be incompatible with the demands of a particular learning task. For example, McDonald and White (1993) have shown that lesions of the hippocampal formation can improve performance on a task that is dependent on the cortico-striatal system.

We report three experiments that further explore the interaction between the cortico-striatal system and hippocampal system. To do this, we used a probabilistic multiple odor discrimination task. The subjects were required to simultaneously solve three odor discrimination problems: A (80\%) vs. B (20\%), C $(67 \%)$ vs. $\mathrm{D}(33 \%)$, and $\mathrm{E}(67 \%)$ vs. $\mathrm{F}(33 \%)$, where the probability that the choice response would be rewarded is in parenthesis. Note that any given choice can result in a rewarded or nonrewarded outcome. Thus, the outcome of any one trial does not perfectly specify the outcome a response will produce when the animal is given the same choice on a later trial. The corticostriatal system that sums the outcome of many trials will even-

\footnotetext{
${ }^{1}$ Corresponding author.
}

E-mail jrudy@clipr.colorado.edu; fax (303) 492-2967.

Article is online at http://www.learnmem.org/cgi/doi/10.1101//m.754208. tually lead to choices that roughly match the real probabilities. However, information captured by the hippocampal episodic memory system then might be incompatible with optimally successful performance.

To evaluate this hypothesis, we used muscimol, a GABA A agonist, to temporarily inactivate either the dorsal striatum or dorsal hippocampus (see Fig. 1 for cannular location for all experiments). The first experiment revealed that inactivating the dorsal striatum impaired performance on the probabilistic discrimination task. In contrast, the second experiment revealed that inactivating the dorsal hippocampus slightly improved performance on this task. In the third experiment we found that inactivating the hippocampus also improved performance on a deterministic discrimination task, A (100\%) vs. B (0\%).

In the first experiment, we assessed the effect of muscimol injections in the dorsal striatum on the performance of the probabilistic reinforcement task. Rats were first pre-trained on a deterministic simple discrimination $(\mathrm{X}+\mathrm{Y}-)$. The goal was to acquaint the rats with the training contingencies in order to reduce training time on the probabilistic training. In the training phase, rats were trained on the three probalistic discriminations: A (80\%) vs. B (20\%), C (67\%) vs. D (33\%), and E (67\%) vs. F (33\%). Rats were trained until they reached the learning criterion of $75 \%$ correct performance (which required 3-8 sessions of training). After reaching the criterion, each rat received two identical test sessions in which either muscimol or the vehicle was injected into the dorsal striatum. The test sessions were identical to the training sessions except that they had six additional trials in which the elements of the $\mathrm{C}$ vs. $\mathrm{D}$ and $\mathrm{E}$ vs. $\mathrm{F}$ problems were recombined to create two novel discriminations (i.e., $\mathrm{C}$ vs. $\mathrm{F}$ and $\mathrm{E}$ vs. D). Thus, the entire test set contained five problems, the three original problems, A+ vs. B-, C+ vs. D-, and E+ vs. F-, and the two new combinations, $\mathrm{C}$ vs. $\mathrm{F}$ and $\mathrm{E}$ vs. D. These recombined trials were included to determine whether the striatum is also involved in novel discriminations.

All 12 rats learned the deterministic $\mathrm{X}+\mathrm{Y}-$ discrimination on the pre-training phase achieving an average performance of $86 \%$ correct by the fourth session. Figure 2 shows that muscimol equally impaired performance on the three trained pairs. A 

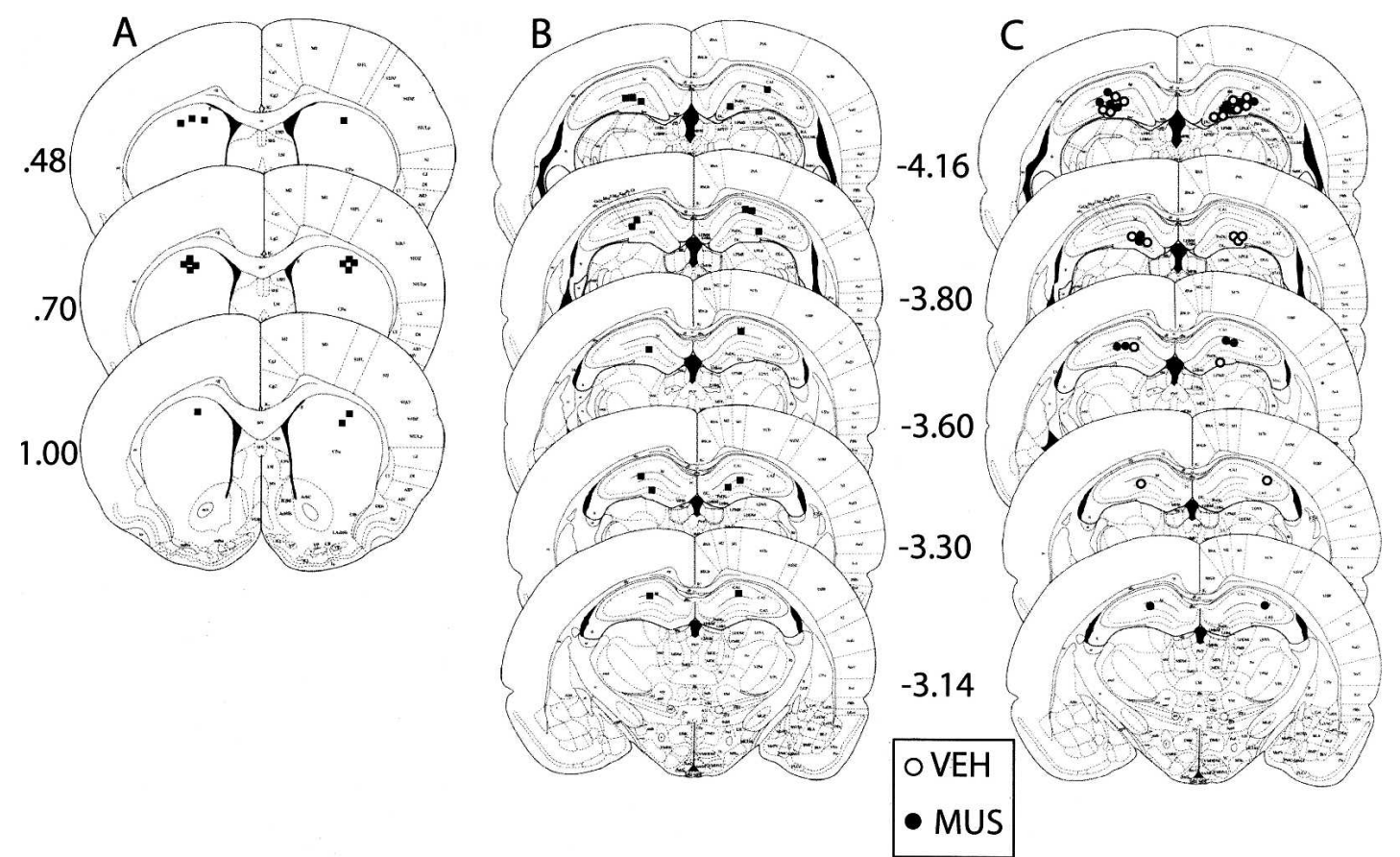

Figure 1. (A) Cannula placements in the striatum in Experiment $1 ;(B)$ cannnula placement in the hippocampus in Experiment 2; $(C)$ cannula placement in the hippocampus in Experiment 3. Numbers indicate the anteroposterior stereotaxic coordinates of the sections relative to bregma. Atlas templates were adapted from Paxinos and Watson (1998).

within-subject repeated measures ANOVA was used to analyze the effects of the drugs in the trained pairs performance (Fig. 2A). The analysis revealed a main effect of trained pair, $F_{(2,11)}=4.84$, $P<0.05$. There was also a main effect of drug, $F_{(1,11)}=36.64$, $P<0.001$. On average, the muscimol injection decreased performance by $13 \%$. There was no interaction between the pair and drug variables, $F_{(2,22)}=0.09, P>0.1$.

The test with the recombined test pair (C vs. F and E vs. D) revealed that the rats' performance was determined by the reward history associated with the elements when rats were injected with the vehicle. They chose the previously rewarded element. However, muscimol significantly reduced the rats' preference for the previously rewarded elements, $t_{(1,18)}=2.89$, $P<0.001$ (Fig. 2B).
A Trained Pairs

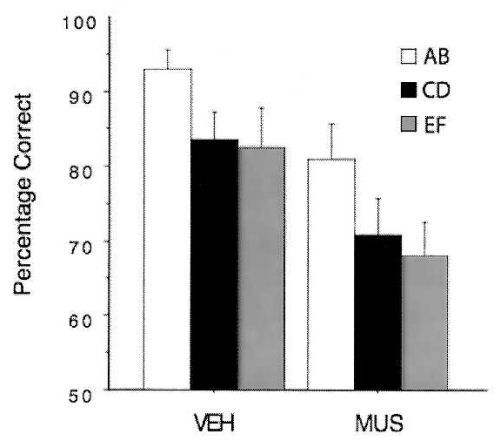

Drug Infusion
B Recombined Pairs Cvs.F and Evs.D

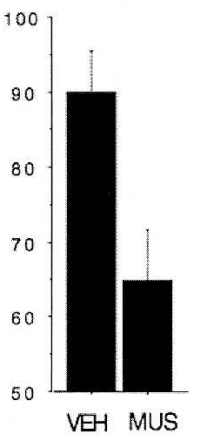

Figure 2. The effect of vehicle and muscimol injections in the dorsal striatum on probabilistic performance. $(A)$ Muscimol impaired performance on the trained pairs $(\mathrm{A}+\mathrm{B}-, \mathrm{C}+\mathrm{D}-, \mathrm{E}+\mathrm{F}-)$. (B) Muscimol impaired performance on the recombined pairs ( $C$ vs. $F, E$ vs. D).
It is possible that information captured by the hippocampal system is incompatible with that contained in the cortico-striatal system. For example, if the rat was recently reinforced for choosing the odor in the left choice position and on the next trial the correct choice was on the right, then the hippocampal system might bias the subject to incorrectly choose the odor on the left. The goal of the second experiment was to evaluate this hypothesis.

All 10 rats learned the deterministic $\mathrm{X}+\mathrm{Y}-$ discrimination on the pre-training phase achieving an average performance of $88 \%$ by the fourth session. Figure $3 \mathrm{~A}$ shows that performance on each trained probabilistic pair improved when mucimol was injected into the dorsal hippocampus. A within-subject repeated measures ANOVA revealed only a main effect of drug, $F_{(1,9)}=11.42, P<0.01$. On average, the muscimol injection improved performance by $7 \%$. The size of this effect may have been limited by the fact that the performance under muscimol was very close to the ceiling ( $94 \%$ correct).

Figure 3B presents the results of the test with the novel test pair (C vs. F and E vs. D). It shows that choice performance was the same whether the rats were tested following an injection of the vehicle or following an injection of muscimol into the dorsal hippocampus $(P>0.1)$.

The results of the second experiment provided some support for the hypothesis that the information captured by the dorsal hippocampus is incompatible with the information captured by the striatum. To further explore this hypothesis rats were trained on a single deterministic discrimination problem, A (100\%) vs. B $(0 \%)$. This problem was used to determine if the hippocampus only impairs performance when the problems are probabilistic.

Rats received a daily 20-trial session for four consecutive days. On the third session, either muscimol $(n=9)$ or vehicle ( $n=10)$ was injected into the dorsal hippocampus. All rats then received a drug-free fourth session. The details of the procedure are identical to those of the pre-training phase described in the Materials and Methods section. A between-subject design was used in this experiment. 


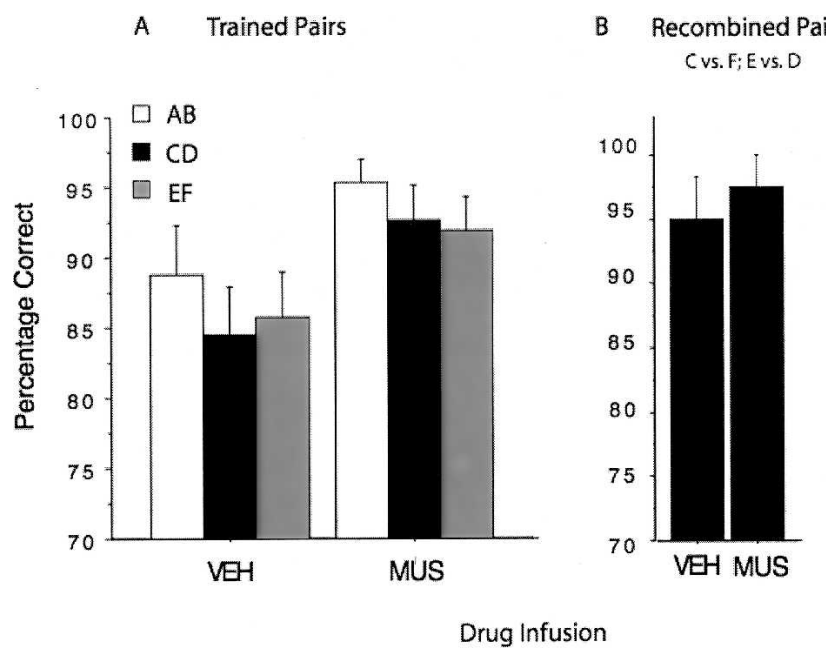

Figure 3. The effect of vehicle and muscimol injections in the dorsal hippocampus on probabilistic performance. $(A)$ Muscimol improved performance on the trained pairs. $(B)$ Muscimol did not have a significant effect on the performance of the recombined pairs.

Rats injected with muscimol displayed enhanced choice performance relative to those injected with the vehicle (Fig. 4). This figure also shows that during the subsequent drug-free session, rats that had been injected with muscimol were impaired relative to those injected with the vehicle. In order to statistically test for group differences on the session of the injection (S3) and the subsequent session (S4), separate ANCOVAs were conducted for performance on each session with group as the between-subject variable and performance on S1 and S2 as covariates. The ANCOVA for S3 performance revealed that muscimol produced a significant improvement relative to the control group, $\left(F_{(1,16)}=\right.$ $6.23, P<0.05)$. The ANCOVA for $S 4$ revealed the opposite effect, a significant impairment of muscimol group relative to the control group, $F_{(1,16)}=5.71, P<0.05$.

Performance on the probabilistic discrimination problems required the contribution of the dorsal striatum. Inactivating this region impaired performance on both the trained problems and on novel test problems constructed by recombining the elements of the already trained problems. This impairment is similar to the one we previously reported on a deterministic task (Atallah et al. 2007).

It is possible that this impairment was due to its effect on the animal's motivation and/or because it produced a motor impairment. Several informal observations are inconsistent with this interpretation. The rats displayed no obvious or subtle motor or motivational problems. They appeared as coordinated and made their choice responses as quickly as controls. Moreover, they never failed to consume the Fruit Loop reward. In a previous study (Atallah et al. 2007), we showed that the response latencies of rats injected with muscimol in the dorsal striatum are similar to control animals. Thus, the overall pattern of results favor the interpretation previously put forth by Atallah et al. (2007) - that rats injected with muscimol were deprived of the processes needed to translate the previously acquired information into correct performance. This conclusion is also supported by anatomical studies that reveal that the part of the dorsal striatum targeted in our experiments projects to cortical area AGm, which is the equivalent of the premotor cortex in primates (Reep et al. 2003).

There is other evidence that ventral striatal areas are involved in acquiring information regarding the value of individual olfactory cues. Viaud and White (1989) showed that postsession amphetamine injections in the ventrolateral striatum im- pair the acquisition of a conditioned emotional response to an olfactory stimulus. Similarly, disruption of the nucleus accumbens (i.e., ventromedial striatum) interferes with the acquisition of new conditioned responses (Hernandez et al. 2002; Atallah et al. 2007). The combined evidence suggests a role for both the medial and lateral parts of the ventral striatum in the acquisition of new behavioral responses to environmental stimuli even though the unique contribution of each structure is unclear.

Overall, it is possible that ventral and dorsal striatum support processes that are necessary for the acquisition and expression of new behaviors, respectively. We have previously suggested two potential pathways through which ventral and dorsal striatal areas can communicate in order for learned information to influence behavioral performance (Atallah et al. 2007). One possibility is that the cortex acts as a link between ventral and dorsal striatum. The other possibility is that the ventral striatum can bias the activity of the dorsal striatum by controlling the release of dopamine in the latter structure.

In contrast, inactivating the dorsal hippocampus improved choice performance. This observation provides another instance in which the memory content of the hippocampal system interferes with performance (see Packard et al. 1989; McDonald and White 1993; Schroeder et al. 2002). McDonald and White (1993) showed that lesion of the fornix, which causes a hippocampal dysfunction, improves on a win-stay discrimination task in the radial maze. Unlike our manipulation, the lesion was performed in the anterograde direction (i.e., the lesion was performed before training). Interestingly, there is some evidence that retrograde hippocampal lesions (performed after training) cause deficits rather than improvements on discrimination tasks (Driscoll et al. 2005; McDonald et al. 2007). Thus, our retrograde pharmacological manipulation replicates the effect of anterograde but not retrograde lesions.

There are a number of differences between those studies and the present one that can account for the discrepancy (e.g., amount of training prior to the manipulation, the modality of the conditioned stimulus). One possibility mentioned by McDonald et al. (2007) is especially noteworthy. Specifically, the hippocampal lesion may have a distal effect on other areas (e.g., striatum, cortex) that are involved in the storage or expression of the acquired information. Those effects may be mitigated if the lesion is performed prior to training or if the hippocampus is only pharmacologically inactivated (Schroeder et al. 2002).

The hippocampus is thought to capture the content contained in single episodes (Teyler and DiScenna 1986; McClelland

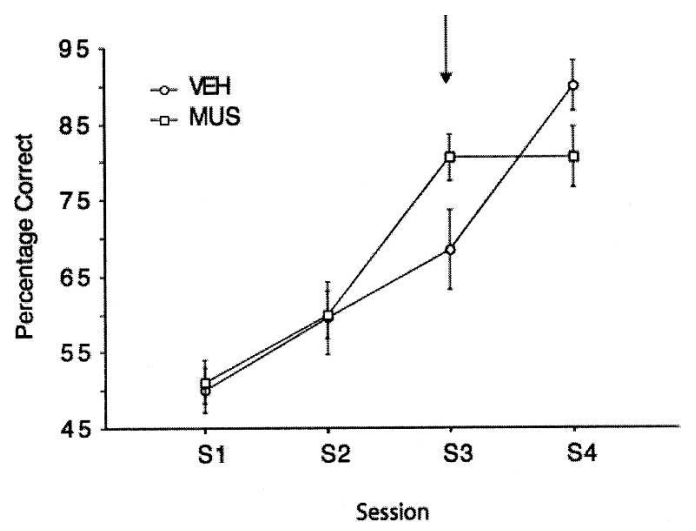

Figure 4. The effect of vehicle and muscimol injections in the hippocampus on a deterministic task. Two groups of animals received either vehicle or muscimol on the third session of training. Muscimol improved performance on the day of the injection, but resulted in a deficit in performance on the session subsequent to the injection (S4). 
et al. 1995; O'Reilly and Rudy 2001; Teyler and Rudy 2007). Depending on the content of an episode, the information the hippocampus captures may be compatible or incompatible with task performance. The present results imply that the hippocampus was capturing trial specific information that was incompatible with the solution being generated by the striatum. Below, we speculate on how this can happen when the cortico-striatal system and the hippocampal system are both involved in the task.

Simultaneous discriminations such as those used in our experiments can be conceptualized as containing several dimensions. We can identify at least three dimensions that are present in our task, odor $\left(\mathrm{O}_{1}\right.$ and $\left.\mathrm{O}_{2}\right)$, spatial location of the goal boxes $\left(\mathrm{L}_{1}\right.$ and $\left.\mathrm{L}_{2}\right)$, and response trajectory $\left(\mathrm{R}_{\mathrm{L}}\right.$ and $\left.\mathrm{R}_{\mathrm{R}}\right)$. In our experiments odor was the relevant dimension (i.e., $\mathrm{O}_{1+}$ vs. $\mathrm{O}_{2}$ ). Now consider a trial early in training in which $\mathrm{O}_{1}$ is located in the goal box positioned at $\mathrm{L}_{1}$. If the animal makes the correct choice, then the values $\left(\mathrm{L}_{1}, \mathrm{O}_{1}, \mathrm{R}_{\mathrm{L}}\right)$ will be associated with reward. Now suppose that on the next trial $\mathrm{O}_{1}$ is in the $\mathrm{L}_{2}$ position. Since the hippocampus captured the information contained in the Trial $N$ episode, including the task irrelevant information, its contribution to choice performance on Trial $N+1$ may lead to an incorrect choice. Given that the striatum is also capturing and accumulating information over training trials, the impact of this irrelevant information, however, should diminish with training. This is because over trials the relevant $\mathrm{O}_{1}$-approach association supported by the striatum will become sufficiently strong to negate the impact of the irrelevant information maintained in the hippocampus.

It is also possible that during training on problems where the hippocampus misguides performance, there are processes that actively take the hippocampus offline. Poldrack et al. (1999) have reported results that are consistent with this idea. They used fMRI to measure activity in the hippocampus in human subjects learning a probabilistic classification task somewhat similar to the one we used with rats and reported that as training progressed, the signal from the hippocampus diminished.

This general idea also provides a way of explaining one of the results obtained in Experiment 3. Recall that when the hippocampus was inactivated prior to Session 3 the rats' performance increased. In addition, however, when these animals were tested drug free in Session 4, they performed slightly worse than the control animals. It is possible that this difference reflects the fact that training during Session 3 contributed to taking the hippocampus offline in control animals, but because the hippocampus was pharmacologically inactivated for the other animals, this did not happen. So, on Session 4 when the hippocampus was active, the information it captured interfered with performance.

The ideas that (1) both the cortico-striatal system and the hippocampal system are capturing information in parallel, and (2) the hippocampal system can go offline, also relate to the results reported by Packard and McGaugh (1996). They used a plus maze that could be solved either by the rat going to the spatial location of a particular arm or by making a particular response, such as turning right. Rats need the hippocampus to learn the spatial location of the correct arm but not for learning a particular response. Note that, in this case, during training the output of the hippocampal system is compatible with the output of the cortico-striatal system. When Packard and McGaugh tested their rats by changing the start location, the results depended on the amount of training. The behavior of rats tested after limited training indicated that they were going to the correct spatial location. However, when they were tested after more extensive training, their performance indicated that they were using the response strategy. These results suggest that the hippocampal output tends to dominate the cortico-striatal output early in training, but the hippocampal system goes offline with extensive training and relinquishes control to the cortico-striatal system.

In conclusion, our results are consistent with the general idea of complementary learning systems in the brain (White and McDonald 2002; Atallah et al. 2004; McDonald et al. 2004), which assumes that the cortico-striatal system and the medial temporal hippocampal system operate in parallel. The information contained in the two systems can either synergize or be incompatible. The experiments we describe uncovered an instance where the information contained in the hippocampal system was incompatible with that provided by the cortico-striatal system. Those results complement findings using very similar learning tasks in human subjects (Knowlton et al. 1996; Poldrack et al. 1999). Future work across multiple levels of analysis and different species will help to further our knowledge of those systems' contribution to learning.

\section{Materials and Methods}

Adult male Long-Evans rats weighing 250-300 g at the start of the experiments were bred at the University of Colorado. Rats were housed in pairs in plastic cages with ad libitum access to water, maintained on a $12 \mathrm{~h}$ light/dark cycle, and given $1 \mathrm{wk}$ to acclimate to colony conditions before experimentation began. Access to food was restricted to maintain the rats at $80 \%$ of their free-feeding weights. To maintain that weight, rats were fed 10 $15 \mathrm{~g}$ of rat chow after each daily training session. All experiments were conducted in accordance with protocols approved by the University of Colorado Animal Care and Use Committee.

Under halothane anesthesia, animals were placed into a stereotaxic apparatus (David Kopf Instruments), and 26 gauge stainless-steel guide cannulae (Plastics One) were implanted bilaterally into the dorsal striatum (DS) or dorsal hippocampus (DH). Based on the rat brain atlas of Paxinos and Watson (1998), the following coordinates were used for bilateral DS implantation: anteroposterior $(\mathrm{AP}),+0.5 \mathrm{~mm}$; mediolateral $(\mathrm{ML}), \pm 3.0 \mathrm{~mm}$; dorsoventral (DV), $-3.6 \mathrm{~mm}$ relative to bregma. In Experiment 2, cannulae were implanted bilaterally into the $\mathrm{DH}(\mathrm{AP},-3.5 \mathrm{~mm}$; $\mathrm{ML}, \pm 5.5 \mathrm{~mm}$; DV,$-3.0 \mathrm{~mm}$ relative to bregma). Cannulae were fixed to the skull with dental acrylic and three small screws. To maintain patency, after surgery, an obturator was placed into the guide cannula that extended $0.5 \mathrm{~mm}$ beyond the tip of the guide cannulae. Rats were allowed to recover for $7 \mathrm{~d}$ before being trained on the behavioral task.

The animals were trained in a Y-shaped Plexiglas maze that contained two goal boxes (for details, see Atallah et al. 2007). The position of each box (i.e., left or right side) was interchangeable on a trial-by-trial basis. The odors were a mix of a food spice and construction sand (100 g, Quirkrete no.1961) and they were spread out across the whole floor of each box. The concentrations ( $\mathrm{g}$ of spice per $\mathrm{g}$ of sand) were selected as follows to equate odor intensity that was subjectively evaluated by the experimenter: cumin (McCormick, $0.17 \mathrm{~g} / 100 \mathrm{~g}$ ), cocoa (Hershey's, 2 $\mathrm{g} / 100 \mathrm{~g}$ ), paprika (McCormick, $1 \mathrm{~g} / 100 \mathrm{~g}$ ), cinnamon (Safeway, 2 $\mathrm{g} / 100 \mathrm{~g}$ ), basil (McCormick, $2 \mathrm{~g} / 100 \mathrm{~g}$ ), garlic (McCormick, 2 $\mathrm{g} / 100 \mathrm{~g})$, anise (McCormick, $2 \mathrm{~g} / 100 \mathrm{~g}$ ), and celery (Spice Islands, $2 \mathrm{~g} / 100 \mathrm{~g})$. The reward was the manual delivery of half a piece of Froot Loop (Kellogg's) through a hole in the top of the box.

Atallah et al. (2007) have described the habituation phase of the experiment. Surgery took place following the habituation phase and the rats were allowed $7 \mathrm{~d}$ to recover before pre-training was initiated. The pre-training phase consisted of a daily session of 20 trials for four consecutive days (a total of 80 trials). During this phase, two spices were used. For half the rats, basil was stimulus $\mathrm{X}+$ and celery was stimulus $\mathrm{Y}-$. For the other half, celery was $\mathrm{X}+$ and basil was $\mathrm{Y}-$ (for details, see Atallah et al. 2007).

Three novel pairs of stimuli were used during training: $\mathrm{A}+\mathrm{B}-, \mathrm{C}+\mathrm{D}-, \mathrm{E}+\mathrm{F}-$. The correspondence between a stimulus and a specific spice was changed across different rats. The spices used in this phase were: cumin, paprika, cinnamon, garlic, anise, 
and cocoa. The overall procedure was similar to the pre-training phase but with a few notable differences: (1) Each session included 45 pseudorandomly distributed trials (15 trials per pair). (2) Reward was scheduled probabilistically. For the A+B - pair, stimulus A was rewarded on $80 \%$ of the trials, whereas stimulus B was rewarded on the remaining 20\%. The reward schedule for both the $\mathrm{C}+\mathrm{D}-$ and $\mathrm{E}+\mathrm{F}-$ pairs was $67 \%$ vs. $33 \%$. A correct response was recorded when the rat approached the stimulus with the higher probability of reward (even if it wasn't rewarded on a specific trial). (3) Before each trial, a piece of Froot Loops was placed at the choice point between the two boxes. The reason was to force the rat to stop and sample both stimuli before making a response. The rat still earned an extra piece of food for making a correct response. Rats were trained once daily until they reached a criterion of $75 \%$ correct performance (averaged across all three pairs).

The test phase consisted of two consecutive daily sessions (49 trials each) performed on two consecutive days using the same overall procedure described above. In addition to the trained pairs (45 trials), four novel trials were added (trial nos. 7 , $12,34,45)$. The novel pairs were recombinations of the previously trained stimuli: CF and ED (two trials of each pair). For those pairs, each stimulus was rewarded on one of two trials. The goal was to ensure that the rats didn't learn about the novel pairs across the two test sessions.

To inject muscimol, rats were gently wrapped in a soft towel, the obturator was removed, and a 33-gauge microinjector (Plastics One) attached to polyethylene 50 (PE50) tubing was inserted through the indwelling guide cannular. The distal end of the PE50 tubing was attached to a $100-\mu \mathrm{L}$ syringe (Hamilton) that was attached to a microinjection unit (model 5000; David Kopf Instruments) that accurately dispensed the desired volume. The microinjector extended $0.5 \mathrm{~mm}$ beyond the tip of the guide cannulae. In Experiments 1 and 2, each rat received a muscimol injection on one session of the test phase and a saline injection on the other. The order of injections was randomly assigned for each rat and counterbalanced across each group.

Muscimol, a GABA agonist provided by Sigma was dissolved in a saline solution. For DS injections, a concentration of 0.25 $\mu \mathrm{g} / \mu \mathrm{L}$ was used. A volume of $0.6 \mu \mathrm{L}$ was injected over $2.5 \mathrm{~min}$ for a total of $156 \mathrm{ng}$ per side. The microinjectors remained in place for an extra $1.5 \mathrm{~min}$. The rats were tested $15 \mathrm{~min}$ after the injection. For DH injections, a muscimol concentration of $0.5 \mu \mathrm{g} / \mu \mathrm{L}$ was used. A volume of $0.5 \mu \mathrm{L}$ was injected over $2 \mathrm{~min}$ for a total of $250 \mathrm{ng}$. The rats were tested an hour after the injection. For both groups, control injections consisted of a saline vehicle using the same parameters for injecting the drug.

At the completion of the experiment, animals were anesthetized with Nembutal $(50 \mathrm{mg} / \mathrm{kg})$ and decapitated; their brains were removed and frozen in cold isopentane. Coronal sections (40 $\mu \mathrm{m}$ thick) were taken through the striatum or hippocampus with a cryostat at $-19^{\circ} \mathrm{C}$, and every third section was mounted. Sections were stained with cresyl violet and examined by light microscopy to visually verify the placement of each cannulae in the DS or DH. Only rats with proper cannula placements were included in the analyses of each experiment (see Fig. 1 for placement).

\section{Acknowledgments}

This research was supported by National Institutes of Health grant NIH RO1 MH61316 to J.W.R.

\section{References}

Atallah, H.E., Frank, M.J., and O'Reilly, R.C. 2004. Hippocampus, cortex, and basal ganglia: Insights from computational models of complementary learning systems. Neurobiol. Learn. Mem. 82: $253-267$.
Atallah, H.E., Lopez-Paniagua, D., Rudy, J.W., and O'Reilly, R.C. 2007. Separate neural substrates for skill learning and performance in the ventral and dorsal striatum. Nat. Neurosci. 10: 126-131.

Driscoll, I., Howard, S.R., Prusky, G.T., Rudy, J.W., and Sutherland, R.J. 2005. Seahorse wins all races: Hippocampus participates in both linear and non-linear visual discrimination learning. Behav. Brain Res. 164: 29-35.

Hernandez, P.J., Sadeghian, K., and Kelley, A.E. 2002. Early consolidation of instrumental learning requires protein synthesis in the nucleus accumbens. Nat. Neurosci. 5: 1327-1331.

Hirsh, R. 1974. The hippocampus and contextual retrieval of information from memory: A theory. Behav. Biol. 12: 421-444.

Knowlton, B.J., Mangels, J.A., and Squire, L.R. 1996. A neostriatal habit learning system in humans. Science 273: 1399-1402.

McClelland, J.L., McNaughton, B.L., and O'Reilly, R.C. 1995. Why there are complementary learning systems in the hippocampus and neocortex: Insights from the successes and failures of connectionist models of learning and memory. Psychol. Rev. 102: 419-457.

McDonald, R.J. and White, N.M. 1993. A triple dissociation of memory systems: Hippocampus, amygdala, and dorsal striatum. Behav. Neurosci. 107: 3-22.

McDonald, R.J., Hong, N.S., and Devan, B.D. 2004. The challenges of understanding mammalian cognition and memory-based behaviours: An interactive learning and memory systems approach. Neurosci. Biobehav. Rev. 28: 719-745.

McDonald, R.J., King, A.L., Wasiak, T.D., Zelinski, E.L., and Hong, N.S. 2007. A complex associative structure formed in the mammalian brain during acquisition of a simple visual discrimination task: Dorsolateral striatum, amygdala, and hippocampus. Hippocampus 17: 759-774.

Mishkin, M. and Petrie, H.L. 1984. Memories and habits: Some implications for the analysis of learning and retention. In Neuropsychology of memory (eds. L.R. Squire and N. Butters), pp. 287-296. Guilford, New York.

Mishkin, M., Malamut, B., and Bachevalier, J. 1984. Memories and habits: Two neural systems. In Neurobiology of learning and memory (eds. G. Lynch et al.), pp. 65-77. Guilford, New York.

Oberg, R.G. and Divac, I. 1975. Dissociative effects of selective lesions in the caudate nucleus of cats and rats. Acta Neurobiol. Exp. 35: $647-659$.

O'Reilly, R.C. and Rudy, J.W. 2001. Conjunctive representations in learning and memory: principles of cortical and hippocampal function. Psychol. Rev. 108: 311-345.

Packard, M.G. and McGaugh, J.L. 1996. Inactivation of hippocampus or caudate nucleus with lidocaine affects expression of place and response learning. Neurobiol. Learn. Mem. 65: 65-72.

Packard, M.G., Hirsh, R., and White, N.M. 1989. Differential effects of fornix and caudate nucleus lesions on two radial maze tasks: Evidence for multiple memory systems. J. Neurosci. 9: 1465-1472.

Paxinos, G. and Watson, C. 1998. The rat brain in stereotaxic coordinates. 4th ed. Academic Press, San Diego.

Poldrack, R.A., Prabhakaran, V., Seger, C.A., and Gabrieli, J.D. 1999. Striatal activation during memory acquisition of cognitive skill. Neuropsychology 13: 564-574.

Reep, R.L., Cheatwood, J.L., and Corwin, J.V. 2003. The associative striatum: Organization of cortical projections to the dorsocentral striatum in rats. J. Comp. Neurol. 467: 271-292.

Schroeder, J.P., Wingard, J.C., and Packard, M.G. 2002. Post-training reversible inactivation of hippocampus reveals interference between memory systems. Hippocampus 12: 280-284.

Sherry, D.F. and Schacter, D.L. 1987. The evolution of multiple memory systems. Psychol. Rev. 94: 439-454.

Teyler, T.J. and DiScenna, P. 1986. The hippocampal memory indexing theory. Behav. Neurosci. 100: 147-154.

Teyler, T.J. and Rudy, J.W. 2007. The hippocampal indexing theory and episodic memory: Updating the index. Hippocampus 17: 1158-1169.

Tulving, E. and Markowitsch, H.J. 1998. Episodic and declarative memory: Role of the hippocampus. Hippocampus 8: 198-204.

Viaud, M.D. and White, N.M. 1989. Dissociation of visual and olfactory conditioning in the neostriatum of rats. Behav. Brain Res. 32: 31-42.

White, N.M. and McDonald, R.J. 2002. Multiple parallel memory systems in the brain of the rat. Neurobiol. Learn. Mem. 77: 125-184.

Received August 27, 2007; accepted in revised form February 21, 2008. 


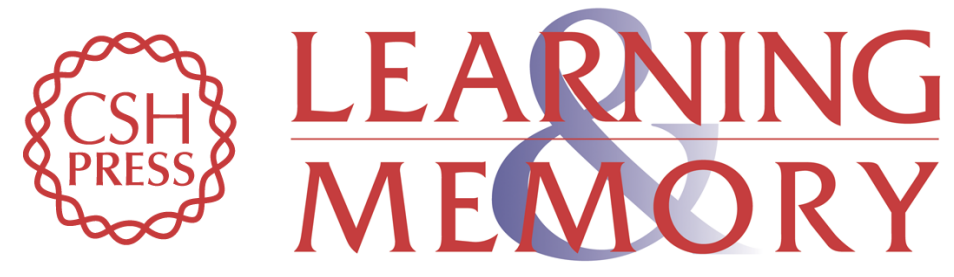

\section{The role of the dorsal striatum and dorsal hippocampus in probabilistic and deterministic odor discrimination tasks}

Hisham E. Atallah, Jerry W. Rudy and Randall C. O'Reilly

Learn. Mem. 2008, 15:

Access the most recent version at doi:10.1101//m.754208

References This article cites 23 articles, 2 of which can be accessed free at:

http://learnmem.cshlp.org/content/15/5/294.full.html\#ref-list-1

License

Email Alerting Receive free email alerts when new articles cite this article - sign up in the box at the Service top right corner of the article or click here. 